\title{
FINGERS: Exploiting Fine-Grained Parallelism in Graph Mining Accelerators
}

\author{
Qihang Chen \\ chenqh19@mails.tsinghua.edu.cn \\ Institute for Interdisciplinary \\ Information Sciences, \\ Tsinghua University \\ Beijing, China
}

\author{
Boyu Tian \\ tby20@mails.tsinghua.edu.cn \\ Institute for Interdisciplinary \\ Information Sciences, \\ Tsinghua University \\ Beijing, China
}

\author{
Mingyu Gao \\ gaomy@tsinghua.edu.cn \\ Institute for Interdisciplinary \\ Information Sciences, \\ Tsinghua University \\ Beijing, China
}

\begin{abstract}
Graph mining is an emerging application of high importance and also with high complexity, thus requiring efficient hardware acceleration. Current accelerator designs only utilize coarse-grained parallelism, leaving large room for further optimizations. Our key insight is to fully exploit fine-grained parallelism to overcome the existing issues of hardware underutilization, inefficient resource provision, and limited single-thread performance under imbalanced loads. Targeting pattern-aware graph mining algorithms, we first comprehensively identify and analyze the abundant fine-grained parallelism at the branch, set, and segment levels during search tree exploration and set operations. We then propose a novel graph mining accelerator, FINGERS, which effectively exploits these multiple levels of fine-grained parallelism to achieve significant performance improvements. FINGERS mainly enhances the design of each single processing element with parallel compute units for set operations, and efficient techniques for task scheduling, load balancing, and data aggregation. FINGERS outperforms the state-of-the-art design by $2.8 \times$ on average and up to $8.9 \times$ with the same chip area. We also demonstrate that different patterns and different graphs exhibit drastically different parallelism opportunities, justifying the necessity of exploiting all levels of fine-grained parallelism in FINGERS.
\end{abstract}

\section{CCS CONCEPTS}

- Computer systems organization $\rightarrow$ Special purpose systems; - Hardware $\rightarrow$ Hardware accelerators; • Mathematics of computing $\rightarrow$ Graph algorithms.

\section{KEYWORDS}

hardware acceleration; graph mining; parallelism

\section{ACM Reference Format:}

Qihang Chen, Boyu Tian, and Mingyu Gao. 2022. FINGERS: Exploiting FineGrained Parallelism in Graph Mining Accelerators. In Proceedings of the 27th ACM International Conference on Architectural Support for Programming

Permission to make digital or hard copies of all or part of this work for personal or classroom use is granted without fee provided that copies are not made or distributed for profit or commercial advantage and that copies bear this notice and the full citation on the first page. Copyrights for components of this work owned by others than ACM must be honored. Abstracting with credit is permitted. To copy otherwise, or republish, to post on servers or to redistribute to lists, requires prior specific permission and/or a fee. Request permissions from permissions@acm.org.

ASPLOS '22, February 28 - March 4, 2022, Lausanne, Switzerland

(c) 2022 Association for Computing Machinery.

ACM ISBN 978-1-4503-9205-1/22/02 . \$15.00

https://doi.org/10.1145/3503222.3507730
Languages and Operating Systems (ASPLOS '22), February 28 - March 4, 2022, Lausanne, Switzerland. ACM, New York, NY, USA, 13 pages. https: //doi.org/10.1145/3503222.3507730

\section{INTRODUCTION}

By locating all subgraphs that are isomorphic to certain user-defined patterns in a given graph, graph mining is a powerful analytics tool that is widely used in different domains, ranging from social science $[18,19,26,58]$, bioinformatics $[2,13,40,41]$, to cheminformatics $[15,20,31,46]$. Unlike conventional graph processing $[24,37,42,48,51]$, graph mining is more challenging due to the much higher algorithmic complexity. As a result, both specialized software frameworks [29, 38, 39, 50, 53, 55] and hardware accelerators $[11,30,57]$ were proposed to improve its processing efficiency.

State-of-the-art graph mining algorithms are pattern-aware [38, $39,50]$, in the sense that they compile the user-defined pattern into an optimized execution plan to guide the mining process. The execution is characterized as exploring a search tree starting from each individual vertex in the input graph, and using set intersection and subtraction operations to determine the next candidate vertex to extend on the partially matched subgraph. This paradigm greatly reduces memory footprints and achieves significant speedups over the previous pattern-oblivious approaches [53], and thus is a promising target for further hardware acceleration [4, 11, 30, 47].

However, existing hardware accelerators have not fully and effectively exploited the abundant parallelism in graph mining. The most recent work, FlexMiner [11], only leveraged coarse-grained parallelism among separate search trees rooted at different input graph vertices, and followed a depth-first search (DFS) order to explore each tree. This results in several inefficiencies. First, the hardware processing elements (PEs) are underutilized due to long memory access stalls caused by the intrinsic dependencies in DFS, analogous to long pipeline stalls in conventional processors. Second, by having each PE execute serial tree search, the resources are inefficiently provisioned. The complex control logic and the expensive local caches dominate the area, analogous to expensive out-of-order scheduling overheads in conventional processors. Third, the search trees starting from different vertices may suffer from load imbalance with real-world power-law graphs. This requires us to provide superior single-PE performance to alleviate the serial bottleneck.

To address these issues, our key insight is that we need to additionally exploit the multiple levels of fine-grained parallelism within each individual tree search inside each PE. This allows us to discover more independent workload to tolerate the pipeline stalls (analogous to multi-threading processors). We can also provision 
more compute units to amortize the expensive control and caching modules with better area and power efficiencies (analogous to vector processors), as well as providing better single-PE performance through parallel processing.

In this paper, we first comprehensively identify and analyze the three levels of fine-grained parallelism in pattern-aware graph mining algorithms in addition to the existing coarse-grained parallelism (Section 3). Branch-level parallelism explores different search tree branches simultaneously instead of strictly following DFS. It introduces more independent tasks but also increases memory footprints. Set-level parallelism processes the multiple set operations in each task in parallel and improves data reuse. But different sets vary significantly in terms of sizes and computations. Segment-level parallelism further parallelizes each set operation by dividing each set into non-overlapping segments. However, processing on different segments has irregular dependencies on both input and output, complicating the hardware design.

We next propose a novel graph mining accelerator, FINGERS, which effectively exploits the aforementioned multiple levels of finegrained parallelism to achieve significant performance improvements (Section 4). To our best knowledge, FINGERS is the first graph mining hardware accelerator that systematically optimizes fine-grained parallelism. Besides the coarse-grained parallelism across multiple PEs, FINGERS enhances the design inside each PE with multiple parallel compute units for set operations. We propose a pseudo-DFS order that determines the proper degree of branchlevel parallelism with balanced benefits and overheads. We use novel segment pairing and load balancing methods to distribute workloads onto multiple compute units in a PE to leverage set-level and segment-level parallelism. We also design an efficient result aggregation mechanism that ensures correct communication and collaboration among the compute units. A single type of hardware unit for set intersection is used for all types of operations include (anti-)subtraction. The results are collected from multiple compute units in a bitvector format using bitwise OR.

We extensively evaluate FINGERS using widely used patterns and popular real-world graph datasets (Section 6). We demonstrate that with the same chip area budget, FINGERS outperforms the state-of-the-art design [11] by $2.8 \times$ on average, and up to $8.9 \times$. Such improvements are mostly from the better PE architecture, where the single-PE performance improves by $6.2 \times$ on average and up to $13.2 \times$, at the cost of less than twice of the area. In particular, we find that different patterns and different graphs exhibit drastically different degrees of each fine-grained parallelism. For example, loosely connected patterns result in high set-level and segment-level parallelism, while dense clique listing primarily benefits from branch-level parallelism. This further justifies the necessity of exploiting all levels of fine-grained parallelism in FINGERS.

In summary, our key contributions in this paper include:

- We identify several inefficiencies in the state-of-the-art graph mining accelerators that only make use of coarse-grained parallelism, including hardware underutilization, inefficient resource provision, and limited single-thread performance under imbalanced loads.

- We present and analyze the three additional levels of finegrained parallelism in pattern-aware graph mining, namely branch-level, set-level, and segment-level parallelism.
- We propose a novel graph mining accelerator, FINGERS, that effectively exploits the aforementioned multiple levels of fine-grained parallelism. FINGERS enhances the design inside each PE with parallel compute units for set operations, and efficient techniques for task scheduling, load balancing, and data aggregation.

- We evaluate FINGERS on different patterns and different graphs, compared against the state-of-the-art baseline. FINGERS achieves $2.8 \times$ on average, and up to $8.9 \times$ speedups with the same chip area budget.

- We demonstrate different patterns and graphs exhibit drastically different degrees of each fine-grained parallelism, further justifying the necessity of exploiting all levels of fine-grained parallelism in FINGERS.

\section{BACKGROUND AND MOTIVATIONS}

\subsection{Graph Mining Algorithms and Systems}

Given a graph $\mathcal{G}(V, E)$ and a set of patterns $S_{p}$, the graph mining algorithm tries to find all the subgraphs in $\mathcal{G}$ that are isomorphic to each of the patterns $p \in S_{p}$. Such isomorphic subgraphs are called the embeddings of the pattern $p$. For example, in Figure 1, the tailed triangle pattern $p$ is described by four vertices $\left\{u_{0}, u_{1}, u_{2}, u_{3}\right\}$, and the graph mining algorithm searches in the input graph for all its embeddings, such as $\{2,1,3,5\}$. There are two ways to define subgraphs in $\mathcal{G}(V, E)$. An edge-induced subgraph $\mathcal{G}^{\prime}\left(V^{\prime}, E^{\prime}\right)$ has vertices $V^{\prime} \subseteq V$ and edges $E^{\prime} \subseteq E$. In contrast, a vertex-induced subgraph $\mathcal{G}^{\prime}\left(V^{\prime}, E^{\prime}\right)$ has vertices $V^{\prime} \subseteq V$ and its edge set $E^{\prime}$ contains exactly those edges in $\mathcal{G}$ whose both endpoints are in $V^{\prime}$.

Graph mining is powerful to solve many graph analytics problems. For example, the most general form subgraph listing, either vertex-induced or edge-induced, aims to list all embeddings of a defined pattern $p$ in a graph $\mathcal{G}$. $k$-clique listing is a special but common case of subgraph listing, which only focuses on the pattern of the size- $k$ clique, i.e., a complete subgraph with $k$ vertices. $k$-motif counting, on the other hand, only counts the number of occurrences for each size- $k$ pattern rather than listing the embeddings. Our hardware supports all these variants.

Graph mining software systems. Previously, various graph mining problems usually used different hand-optimized specialpurpose algorithms, such as for triangle counting [22, 25, 27, 43, 44], clique listing [12, 14, 21], motif counting [1, 35, 45], and subgraph listing [5, 6, 32, 33, 36, 49, 52]. However, it requires substantial programming efforts to hand-tune each algorithm individually for correctness and performance. Recently, many generic graph mining software frameworks have been proposed to improve programmability and also optimize performance in a generally applicable way. Representative examples of such systems include Arabesque [53], GMiner [7], G-thinker [56], RStream [55], Fractal [16], AutoMine [39], GraphZero [38], GraphPi [50], Pangolin [10], Peregrine [29], Sandslash [9], DwarvesGraph [8], and aDFS [54].

The above graph mining frameworks can be classified into two main categories. Early systems are pattern-oblivious (also known as embedding-centric) $[7,10,16,53-56]$. They represent the graph mining problem as a large search tree, whose level $k$ contains all the possible embeddings of size $k+1$ that are extended from the parent level. The tree keeps extending in a breadth-first search (BFS) [53] or 


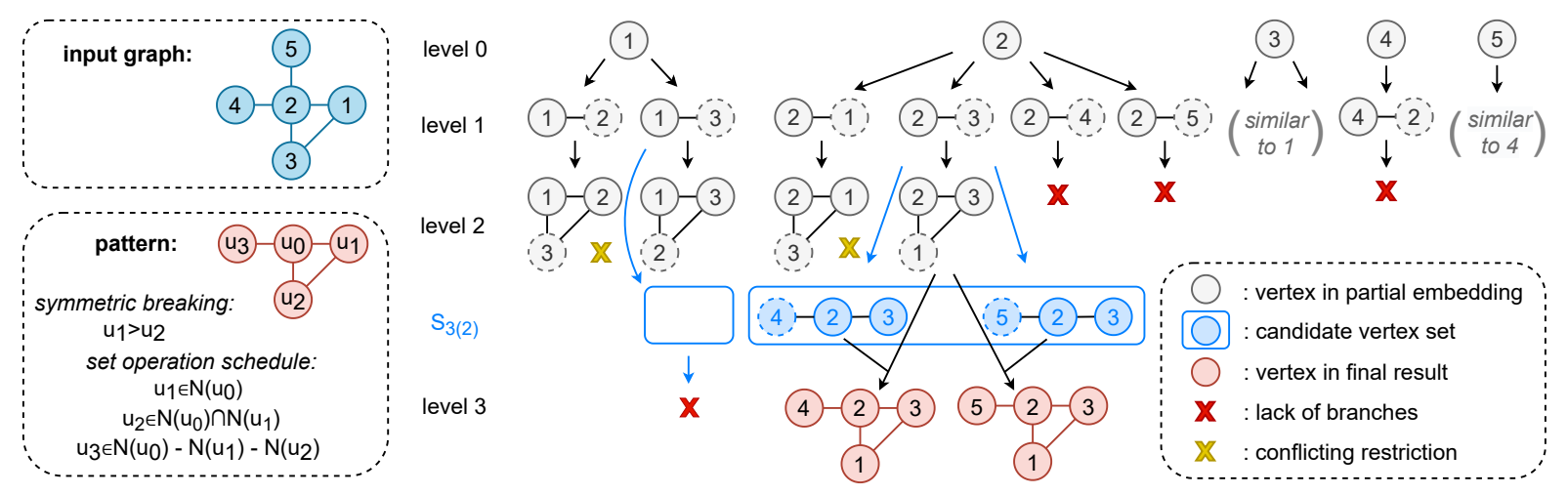

Figure 1: Pattern-aware graph mining algorithm for the tailed triangle pattern.

depth-first search (DFS) [16, 54] manner. At each intermediate level, the partial embeddings that cannot match the pattern could be early pruned. At the leaf level, all final embeddings go through expensive isomorphic checks. This leads to high computation complexity and exponential memory footprint growth.

In contrast, pattern-aware (a.k.a., set-centric) systems exploit the structure of the pattern to first synthesize an efficient execution plan, including the orders and the conditions of adding each new vertex into the candidate embedding $[9,29,38,39,50]$. Following such an optimized plan allows us to greatly reduce the time and space complexity. Our work focuses on these pattern-aware algorithms, which we further introduce in details below.

Pattern-aware graph mining algorithms. Figure 1 uses the tailed triangle pattern with four vertices as an example to illustrate the pattern-aware algorithm [38, 39]. We aim to mine vertexinduced subgraphs for now, and explain edge-induced cases later. Before mining on the input graph, the framework compiles the pattern to generate an execution plan as follows. First, it determines the order of vertices to consider in the pattern [39], which in our example is assumed to be $u_{0}, u_{1}, u_{2}, u_{3}$, i.e., $u_{i}$ is an ancestor of and considered earlier than $u_{j}$ only if $i<j$. Second, for each $u_{i}$ to be added at each step, the compiler generates its set operation schedule $S_{i}$, which describes the candidate vertex set that $u_{i}$ can be mapped to according to the connections to its ancestors. Essentially, $u_{i}$ should be a common neighbor to all connected ancestors, but exclude the neighbors of disconnected ancestors. For example, $S_{3}=N\left(u_{0}\right)-N\left(u_{1}\right)-N\left(u_{2}\right)(N(u)$ denotes the neighbors of $u)$ in Figure 1 because $u_{3}$ is connected to $u_{0}$ but not $u_{1}$ and $u_{2}$. At each step, these set operation schedules will be materialized to concrete candidate vertex sets after the ancestors are determined during the search. Third, the plan also includes symmetric breaking restrictions, if the pattern has non-trivial automorphisms [38]. As in Figure 1, $u_{1}$ and $u_{2}$ are symmetric in the pattern, so exchanging their mapped vertices results in automorphic embeddings. We would like to only count such embeddings once, and also prune the mining process early rather than extending both to the end, to save time and memory usage. To do so, a set of restrictions are applied to the mapped vertex IDs in the input graph, e.g., forcing $u_{1}>u_{2}$. How to compile an optimized execution plan is an extensively studied topic

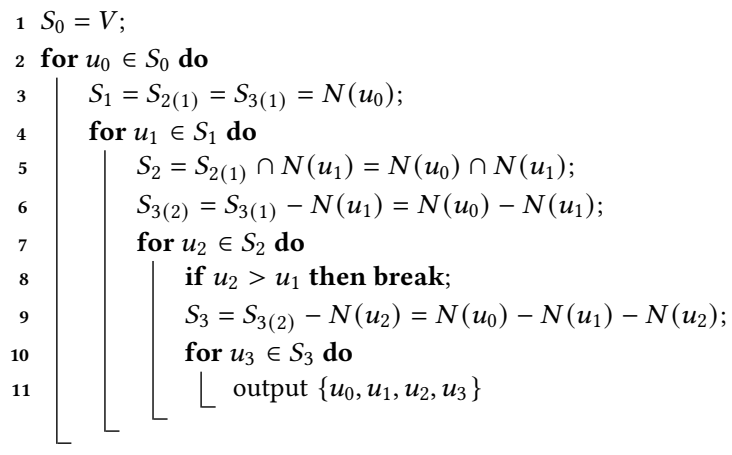

Figure 2: Algorithm for mining the tailed triangle pattern.

in graph mining algorithms $[29,38,39,50]$. Our hardware design is compatible with these proposals as it works with the generic execution plan format described above.

The execution plan guides the mining process on the search tree as shown in Figure 1. Figure 2 represents the algorithm as nested loops following the DFS manner. At each level $i$, we extend the current partial embedding with a new vertex from the input graph, which is mapped to $u_{i}$ in the pattern. This new vertex is chosen from the candidate vertex set materialized from the set operation schedule $S_{i}$, and different choices grow to multiple branches in the next level. For example, if at level 0 we choose $u_{0}=2$, then at level $1, u_{1}$ can be any vertex in $S_{1}=N\left(u_{0}\right)=\{1,3,4,5\}$, corresponding to the four branches 2-1, 2-3, 2-4, 2-5 in Figure 1.

During the tree search, we obtain the concrete candidate vertex sets from the set operation schedules after each vertex is determined. Such materialization is done incrementally, i.e., for the candidate vertex set $S_{j}$ of level $j$, at each previous level $i<j$ we incrementally update a partial result $S_{j(i)}$ as follows.

$$
S_{j(i+1)}= \begin{cases}S_{j(i)} \cap N\left(u_{i}\right) & \text { intersection } \\ S_{j(i)}-N\left(u_{i}\right) & \text { subtraction } \\ N\left(u_{i}\right)-S_{j(i)} & \text { anti-subtraction }\end{cases}
$$


Depending on whether $u_{j}$ is connected to $u_{i}$ or not, intersection or subtraction is used, respectively. Anti-subtraction is used when $u_{j}$ is only connected to $u_{i}$ but none of the previous ancestors. In this case, logically $S_{j(i)}$ stores the negation of the partial candidate vertex set, i.e., the union of all ancestor neighbor lists that should be subtracted from $N\left(u_{i}\right)$ with anti-subtraction. Such union results are quite large and have high memory overheads. In our design, we postpone the actual computation until reaching the first connected ancestor and use multiple anti-subtractions instead.

$S_{j(i)}$ takes into account the connections of already determined ancestors until the current level. All the lower levels will share this same set of ancestors, and thus the partial result can be reused by the entire subtree without recomputing. For example, assume we are on branch 2-3 and at level 2 in Figure 1. We can compute $S_{3(2)}=N\left(u_{0}\right)-N\left(u_{1}\right)=\{4,5\}$ (Figure 2 Line 6). This result can be reused by different instances of $u_{2}$ to obtain the final $S_{3}$ (Figure 2 Line 9). E.g., when $u_{2}=1, S_{3}=S_{3(2)}-N\left(u_{2}\right)=\{4,5\}$, resulting in the final results 2-3-1-4 and 2-3-1-5.

Symmetric breaking restrictions are also applied to prune the search tree at corresponding levels. Figure 2 Line 8 requires $u_{2}<u_{1}$. Therefore although $u_{2}=3 \in S_{2}$ on the branch 2-1, it does not produce a valid embedding because 2-1-3 is automorphic to 2-3-1. The search along it can be immediately terminated, saving compute time and memory space.

Set operations and representation. From Equation (1) we can see that the key computations in graph mining are set operations, including set intersection $(\cap)$ and set subtraction $(-)$. In the above vertex-induced cases, both operations are needed. For edge-induced subgraph mining, however, the set subtractions should be removed, as we do not enforce absence of edges in the subgraphs for exact edge matches. By supporting both set intersection and subtraction, our design is general and works for both scenarios.

In this work, we use a common representation for sets as ordered lists of vertex IDs. Calculating the intersection or difference of two sets then becomes a merge using one-pass sequential scans over the two lists. In this way, the outputs of these operations are still ordered lists. As long as the input graph's original vertex neighbor lists are sorted (potentially after a one-time pre-processing pass), the result of any merge will also be sorted, and the mining process will not require any explicit sort operations. Such merge-based set operations can be efficiently implemented in hardware [11, 47].

Multi-pattern mining. Although we present the above algorithm for a single pattern, it could be easily extended to support mining multiple patterns simultaneously [39]. Basically, if the patterns share some identical components, their search trees can be merged so that the intermediate results for the identical part can be reused. Multi-pattern mining only extends the search tree with more branches, and does not affect the overall computations [11].

\subsection{Existing Hardware Accelerators}

Graph mining algorithms are quite compute-intensive, and mining large-scale real-world graphs may require several hours or days even on modern multi-core processors [39]. As a result, specialized hardware acceleration has recently been applied to graph mining. Gramer [57] was one of the first graph mining accelerators. It used specially designed caches to leverage locality and pinned frequently accessed data on chip. However, Gramer followed the sub-optimal pattern-oblivious paradigm, and the huge performance gap compared to pattern-aware algorithms could not be closed by hardware acceleration, making Gramer even slower than softwarebased systems like AutoMine [39]. More recent designs instead used the better pattern-aware algorithms and focused on efficient set operations. TrieJax [30] used a worst-case-optimal-join algorithm to perform set intersections, but it could not mine vertex-induced subgraphs due to the lack of set subtraction support. IntersectX [47] enhanced conventional general-purpose processors with stream instruction set extensions for efficient set operations. It added a special hardware unit that accepted the two input sets as streams flowing through a numeric comparator for set intersection and subtraction. SISA [4] also designed a special instruction set for set operations on processing-in-memory (PIM) architectures. Our work exploits fine-grained parallelism in graph mining, which was not the main focus of these prior designs.

FlexMiner [11], as a state-of-the-art accelerator design, adopted multiple hardware processing elements (PEs) to exploit the massive amount of coarse-grained parallelism in graph mining. Such coarse-grained parallelism comes from the independent tasks starting from different initial root vertices, i.e., level 0 in Figure 1 and Figure 2 Line 2. Each PE therefore executes DFS on a separate search tree, controlled by a scheduler and an ancestor vertex stack. A global shared cache and per-PE private caches are used to reuse frequently accessed data. We use FlexMiner as our baseline, and further improve its performance.

\subsection{Motivations for Fine-Grained Parallelism}

As described above, FlexMiner leverages coarse-grained parallelism by executing separate search trees on multiple PEs. We consider such a design analogous to the multi-core architecture. While the number of vertices is usually large enough to enable sufficient parallelism for FlexMiner, there are still several inefficiencies.

First, the PEs may be underutilized, due to long memory stalls caused by the intrinsic dependencies between adjacent search tree levels when following the DFS order. For example, for each newly extended vertex $u_{2}$ in Figure 2 Line 7 , the PE needs to fetch its neighbor list $N\left(u_{2}\right)$ from memory to compute the candidate vertex set $S_{3}$ in Line 9. Only after that, the PE can start the next level in Line 10. Essentially, each level must be sequentially explored following DFS. If a neighbor list misses in the caches, the PE has no other work to do, and must stall during the long-latency memory access. This issue is analogous to long pipeline stalls due to accessing dependent data from memory in conventional processors.

Second, the PE design suffers from inefficient resource provision. Each PE in FlexMiner needs complex hardware modules for scheduling the DFS execution and caching data, while the actual compute unit for set operations only takes a small portion of the area. In fact, a FlexMiner PE costs $0.18 \mathrm{~mm}^{2}$ under $15 \mathrm{~nm}$, while a set intersect unit needs less than $0.01 \mathrm{~mm}^{2}$ under $28 \mathrm{~nm}$ in our implementation (Section 6.1). This issue is analogous to expensive out-of-order scheduling overheads in conventional processors.

Third, despite abundant individual initial vertices, solely relying on coarse-grained parallelism still causes load imbalance when processing real-world power-law graphs. Because each individual 
search tree DFS is assigned to a single PE and not further parallelized, the search trees rooted at a few very-high-degree vertices will require much longer time than others, and potentially become the bottleneck. Just as in conventional processors, if a task is dominated by long sequential work, multi-core parallelization can only provide limited benefits.

To address the above issues, our key insight is that we need to additionally exploit the multiple levels of fine-grained parallelism within each individual search tree on a single PE. By discovering more fine-grained independent tasks both at each tree level and across different tree branches, we can effectively utilize the PE and tolerate memory stall overheads, analogous to multi-threading processors. Also, by using multiple compute units in one PE to process the fine-grained tasks in parallel, we can amortize the expensive scheduling logic and cache modules to get better performance per area, using the same philosophy as vector processors. Finally, by parallelizing the search tree DFS inside each PE, we can achieve higher single-thread performance and therefore are able to provide significant speedup even for skewed workloads.

\section{FINE-GRAINED PARALLELISM IN GRAPH MINING}

In this section, we first introduce the key characteristics of the three levels of fine-grained parallelism in pattern-aware graph mining We build our novel accelerator, FINGERS, in the next section, to exploit such fine-grained parallelism for better performance and efficiency.

\subsection{Tree-Level (Coarse-Grained) Parallelism}

In pattern-aware graph mining, different search trees rooted at different initial vertices in the input graph are completely independent and can be processed in parallel. As in Figure 1, the root can be each of the five vertices. This coarse-grained parallelism is the one that FlexMiner leveraged across its multiple PEs [11]. However, as discussed in Section 2.3, purely relying on coarse-grained parallelism is not a perfect solution.

\subsection{Branch-Level Parallelism}

A conventional processor or a FlexMiner PE sequentially processes an individual search tree in the DFS order [11,39]. DFS is efficient for such sequential processing because it reduces the memory consumption for intermediate results during tree traversal. However, the search tree can actually be traversed in any order, either DFS or BFS, without affecting correctness. This means that different branches at each level in the search tree (e.g., 2-1, 2-3, 2-4, 2-5 in Figure 1), which are generated by choosing different vertices mapped to $u_{i}$, can be freely processed in parallel. From another perspective, such branch-level parallelism is equivalent to parallelizing the for loops in Figure 2. Actually, branch-level parallelism is a generalization of the tree-level parallelism, where the latter only parallelizes the root vertex choices, and the former also parallelizes the lower tree levels. aDFS [54] exploited such branch-level parallelism in its software implementation, in order to increase the parallelization opportunities to fully utilize multi-core processors when the tree-level parallelism was limited due to selection constraints on the initial vertex.

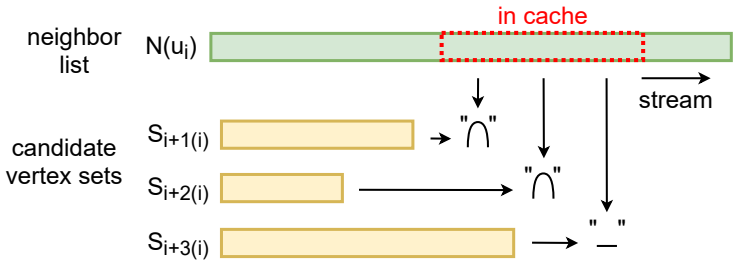

Figure 3: Set-level parallelism improves neighbor list reuse.

Benefits \& challenges. Beyond parallelizing over multiple PEs, our key insight is that branch-level parallelism also enables more available tasks to schedule on each single PE, to tolerate memory access latencies due to dependencies across tree levels in DFS, and thus improves PE utilization. Again consider the example in Figure 1 where we have four branches following the root vertex 2 . If the caches contain the neighbor list of vertex 3 but not that of vertex 1 , we can first schedule branch $2-3$, and at the same time prefetch the neighbor list of vertex 1 to prepare for branch 2-1. This is similar to multi-threading processors that dynamically switch to a readyto-execute context when the current one is stalled.

On the other hand, exploring too many branches simultaneously would greatly increase the intermediate data size, causing cache contention or even using up the memory capacity [55]. Also the workloads on different branches are imbalanced. Hence we must carefully decide the execution order and the degree of branch-level parallelism at each tree level.

\subsection{Set-Level Parallelism}

Within each task of extending a new vertex to the partial embedding, i.e., growing a branch in the search tree, we apply Equation (1) to incrementally materialize the candidate vertex sets for future levels using the neighbor list of the current vertex $u_{i}$. At level $i$, there are at most $k-i$ independent candidate vertex sets $S_{j(i)}, j=i+1, \ldots, k$ that can be updated in parallel, where $k$ is the size of the pattern. For example, Lines 5 and 6 in Figure 2 compute two such sets. Many such sets usually share some common operations. E.g., $S_{1}, S_{2(1)}, S_{3(1)}$ in Figure 2 Line 3 are identical, and we only compute once.

Benefits \& challenges. Besides offering more independent workloads for parallel scheduling, set-level parallelism also improves data reuse. From Equation (1) we see that different sets $S_{j(i)}, j=$ $i+1, \ldots, k$ share the same neighbor list of $u_{i}$ (Figure 2 Lines 5 and 6 ). For a high-degree vertex with a large neighbor list that does not fit in the cache, if these sets are sequentially updated, the neighbor list must be refetched from the memory multiple times. Instead, if the sets are processed in parallel as in Figure 3, we can stream the neighbor list into the cache and fully utilize each chunk for all sets.

Nevertheless, the computations on these sets could differ substantially. Depending on how the vertex is connected to the ancestors, the set updates can be either intersection or (anti-)subtraction, resulting in different workloads. Also, the amount of set-level parallelism $(\leq k-i)$ is limited due to common computations and decreases when getting deeper in the tree, requiring flexible resource allocation for high utilization. 


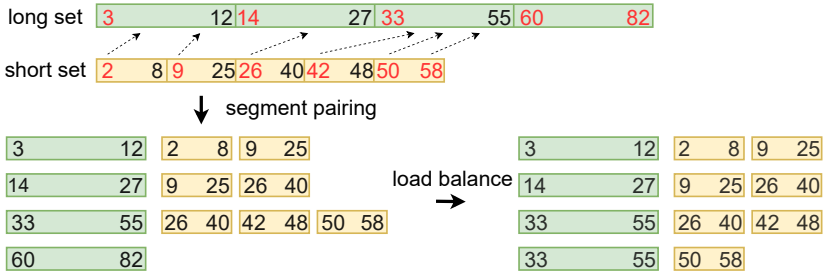

Figure 4: Segment-level parallelism. The irregular segment pairing between the two sets could be alleviated with the load balance strategies described in Section 4.2.

\subsection{Segment-Level Parallelism}

For each set operation, we can divide the two input sets (in the form of ordered lists) each into multiple segments of distinct ranges, apply the operations to each pair of (partially) overlapped segments in parallel, and finally aggregate the results. We observe that in Equation (1), one input is a partially materialized candidate vertex set, and the other is always a neighbor list of a vertex. Because both intersections and subtractions reduce the result set size, usually the first input set $S_{j(i)}$ is shorter, and the second set $N\left(u_{i}\right)$ is longer. We thus call them short and long input sets. To leverage segment-level parallelism, each vertex neighbor list (the long set) is pre-divided into read-only fixed-length segments of size $s_{l}=16$ in our design. The short set is also divided into segments of size $s_{s}=4$ during the computation. Each long segment will be paired with several short segments which are overlapped with it, and the set operations are applied to all such segment pairs, to calculate their intersections or differences through one-pass merge. For example in Figure 4, the two input sets are respectively divided into four and five segments. The first segment $[3,12]$ in the first set overlaps with both of the first two segments $[2,8]$ and $[9,25]$ in the second set. Finally, the results from these segment pairs need to be properly aggregated and merged (see below).

Benefits \& challenges. Segment-level parallelism enables each set operation to be parallelized, which is particularly effective for very-high-degree vertices with huge neighbor lists. Such parallelization can be enabled without changing the upper-level execution order of the search tree, meaning that the parallel units can amortize the other costly logic in the PE, similar to vector processors. Thus the idea of segment-level parallelism has been exploited in SIMD-based software implementations [28]. Additionally, our new insight is that for specialized accelerators, segmenting the sets into similar sizes results in fine-grained individual workloads that can potentially achieve more balanced parallel execution.

However, the challenges here include how to efficiently pair the segments from the two sets with overlapped ranges. Each segment in a set may be paired with zero, one, or multiple segments in the other set as in Figure 4. We need an effective strategy to ensure load balance under potentially irregular workload distribution. Finally, how to aggregate the results into a well-formed ordered list is also non-trivial, especially for set subtraction.

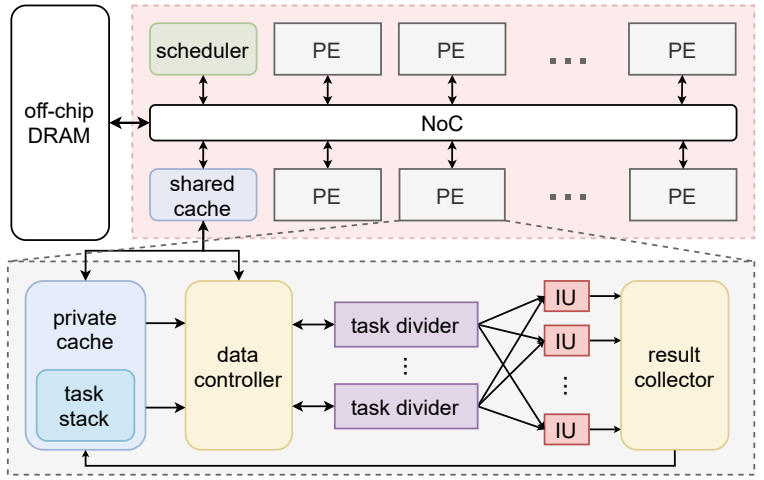

Figure 5: FINGERS architecture overview.

\subsection{Summary of Design Challenges}

To efficiently exploit the aforementioned fine-grained parallelism, we must address the following key challenges. First, we should carefully decide resource allocation among different parallelism levels, i.e., how many branches/sets/segments to process in parallel on the limited number of hardware units. Different allocations lead to tradeoffs between data reuse, intermediate data footprint, and resource utilization. Second, the parallel hardware units must involve effective load balance strategies, as the workloads with different branches/sets/segments are highly irregular due to the diverse vertex degree distribution in input graphs. Third, parallelizing an individual set operation requires special hardware support for communication and cooperation among segments, such as input pairing and output aggregation.

Note that the above three levels of fine-grained parallelism could also be used in software frameworks $[28,54]$. However, parallelizing with increasingly finer workload distribution on general-purpose cores would incur high overheads of thread launching and cooperation, eventually diminishing the return. We thus opt for specialized hardware, and leave software approaches as future work.

\section{FINGERS ARCHITECTURE}

We propose a novel graph mining accelerator, FINGERS, which efficiently exploits the multiple levels of fine-grained parallelism in Section 3 to achieve significant performance improvements. Figure 5 illustrates the overall architecture of FINGERS. FINGERS contains multiple PEs connected through a network-on-chip (NoC). Data are fetched from/to the off-chip DRAM, and buffered in an on-chip shared cache. A global scheduler assigns individual search trees rooted at different vertices in the input graph to separate PEs. The exploration of each tree is entirely done in one PE. Such highlevel architecture is similar to FlexMiner and utilizes the coarsegrained parallelism [11], so they could share the same programming interface and system integration choices.

The key features of FINGERS reside inside each PE, where we further utilize fine-grained parallelism to speed up single-PE processing. The tree search executed on each PE is decomposed into tasks. We define a task as the work to extend a new vertex to the current partial embedding. For example, growing the tree in Figure 1 
from the root vertex 2 to branches 2-1 and 2-3 are two individual tasks; extending 2-3 to 2-3-1 is also a task. Each task thus involves updating multiple candidate vertex sets for the future tree levels. One of these sets becomes a fully materialized candidate vertex set, which is used to spawn new tasks for the next tree level. Consequently, the set and segment levels of parallelism are within each task, while the branch-level parallelism affects how many separate tasks are available to the PE.

Each PE has multiple intersect units (IUs) as the main compute units for various set operations. We use 24 IUs per PE as default, and explore the impact in Section 6.4. We store all candidate vertex sets during the tree search in the PE's private cache, and only spill to the shared cache if they overflow. These sets are always associated with specific tasks, so we can manage them in collaboration with the task stack. On the other hand, the neighbor lists of input graph vertices bypass the PE private cache and are only buffered in the shared cache. Their access patterns are mostly streaming (see Figure 3 ). In addition, such neighbor lists are larger than the candidate vertex sets and thus are harder to cache. Furthermore, our task scheduling can effectively tolerate their fetch latencies.

The PE realizes a 5-stage macro pipeline. (1) We first pop one or more tasks from the stack to schedule, and fetch their candidate vertex sets and vertex neighbor lists from the PE private cache and the shared cache, respectively (Section 4.1). (2) Next, the data controller receives the input sets and generates a head list from each, which contains the first elements (heads) from all segments of the set. During the head list generation, symmetric breaking restrictions are applied to prune entire segments with no valid vertices. (3) The head lists are then sent to the task dividers for segment pairing and load balancing (Section 4.2). The set operations are divided by segments into parallel workloads. (4) The task dividers then issue the workloads to the IUs for intersection or subtraction. (5) Finally, the results from separate IUs for the same set operation are properly aggregated in the result collector (Section 4.3). We apply the symmetric breaking restrictions again to filter invalid vertices in the results. The fully materialized candidate vertex sets become the new tasks and are pushed to the stack.

As FINGERS processes generic search tree structures, it naturally supports multi-pattern mining just like FlexMiner does [11]. The first few tree levels are common, until the point where different patterns diverge to separate tree trunks. The trunks of different patterns are treated similarly to different branches in single-pattern execution, but with additional marks to differentiate their patterns.

\subsection{Resource Allocation and Task Scheduling}

With multiple levels of parallelism, a key question is how to allocate the limited number of IUs in the PE. For example, with 4 IUs, we can process either 4 individual tasks, 4 set operations in one task, 4 segments in a single set operation, or any mix of these. We notice that a single task usually includes multiple set operations on multiple segments, therefore its set- and segment-level parallelism is already abundant. To simplify scheduling, a PE primarily parallelizes each task on its IUs, while sequentially executing different tasks.

To parallelize a task on the multiple IUs, we prioritize set-level parallelism to improve data reuse. We execute all the set operations simultaneously on the IUs, so the neighbor list of the newly

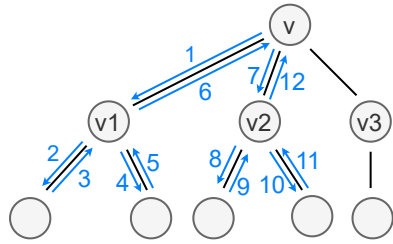

DFS

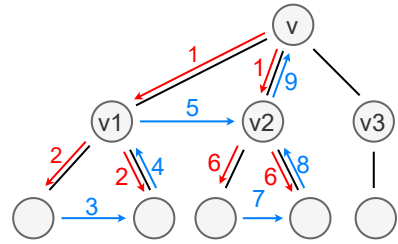

pseudo-DFS
Figure 6: The pseudo-DFS task scheduling order to traverse search trees with appropriate branch-level parallelism.

extended vertex can be shared and maximally reused by all these operations as explained in Figure 3. Usually the number of set operations is smaller than the number of IUs in a PE (24), so each set operation would still use a few IUs, where the segment-level parallelism can be utilized. Because these set operations have diverse workloads, IUs are not uniformly allocated among them, but instead follow the load balance strategies introduced later in Section 4.2.

On the other hand, we do not need to heavily exploit branchlevel parallelism to have multiple tasks execute in parallel. However, maintaining a small number of available tasks is still necessary in order to hide the memory access latency and keep the PE macro pipeline well utilized (Section 3.2). We propose the pseudo-DFS task scheduling order illustrated in Figure 6 as a slightly modified version of DFS, to traverse the tree with appropriate degrees of branch-level parallelism. Specifically, we gather a small number of tasks spawned from the same parent task as a task group before pushing them into the task stack. For example, $v_{1}$ and $v_{2}$ in Figure 6 form a task group, both spawned from the root $v$. The tasks in a group are popped from the stack together. Then we first execute those tasks whose input neighbor lists are already in the shared cache while fetching the others. Such selection is implicitly done without explicitly tracking the cache content. We simply issue all tasks to the cache; hits return immediately to resume the corresponding tasks, while misses cause a task to wait. The branch-level parallelized tasks also share the $S_{j(i)}$ data in the PE private cache. The task group size, i.e., the degree of branch-level parallelism, is a configurable parameter. It is chosen to fully utilize the hardware while preventing too large intermediate data. For simplicity, we set it as the minimum number of tasks to fully occupy the IUs in a PE, where the IU count needed for each task is estimated using the average sizes of the two input sets: vertex neighbor lists and candidate vertex sets. Using average set sizes is good enough, as different tasks may cancel each other's error. We observe that performance is insensitive to these parameters. Overall, pseudo-DFS is able to improve PE resource utilization while not exponentially expanding intermediate data.

\subsection{Segment Pairing and Load Balancing}

To distribute the multiple set operations in a task to the multiple IUs in a PE, the task dividers use the head lists of these sets for segment pairing and load balancing. Recall that a head list contains the first elements (heads) from all segments of the set. Each task divider can match a pair of head lists with up to 15 heads for the long set (corresponding to a vertex neighbor list of size $15 \times s_{l}=240$ ) and up 


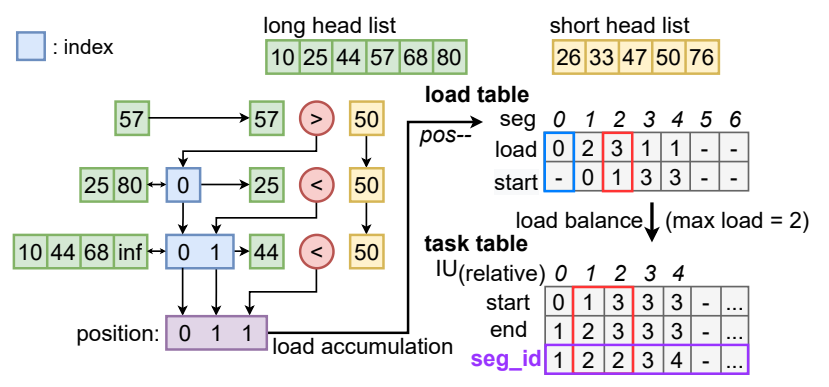

Figure 7: The task divider unit for input segment pairing and load balancing.

to 24 heads for the short set (corresponding to a candidate vertex set of size $24 \times s_{s}=96$ ). Such limits are sufficient for most vertices in real-world graphs. Further increasing the head list sizes would increases the hardware cost. In rare cases with even longer head lists, they can be split into chunks to be matched on multiple task dividers or processed sequentially (see below).

To pair overlapped long and short segments, as in Figure 7, we organize the 15 long heads as a binary tree, and stream in each short head to compare with them. This hardware-based binary search is only used to match among the heads for segment pairing; the actual intersection/subtraction of paired segments uses the merge-based IUs as described in Section 4.3. The resultant index for the $i$ th short head, $\operatorname{pos}_{i}$, indicates the position of a long head immediately larger than it, so the $i$ th short segment should be paired with the long segments from $\operatorname{pos}_{i}-1$ to $\operatorname{pos}_{i+1}-1$ (both included). We use this $\operatorname{pos}_{i}-1$ to index into a load table, and update the corresponding columns. The load table only needs 15 columns and 2 registers per column to store the number and the starting index of the short segments paired with each long segment. Such a small table can be implemented efficiently with separate registers for concurrent accesses.

The task divider next distributes the loads of the long segments to the IUs. We let each IU process one long segment with multiple paired short segments. Segments have fixed lengths, so the load can be described by the number of short segments. Each long segment with its paired short segments can be assigned to one or multiple IUs for load balancing, similar to Figure 4. We first omit any long segment that has no short segments paired with it (load $=0$ in the load table, the blue box in Figure 7) except for anti-subtraction. Using a pre-determined maximum load threshold, we further split the too many short segments paired with the same long segment onto multiple IUs (load $=3>2$ for long segment \#2 in the load table, the red box). The load assignments after balanced are stored into a task table, which records the long segment index and the range of the paired short segments for each IU.

Coordination between multiple task dividers. Each PE has multiple task dividers (default 12 per PE). Each task has multiple set operations to be scheduled to the IUs. Also, some input sets could be quite large in power-law graphs and their head lists must be split into chunks. The task dividers share the same set of physical IUs in the PE, and they issue their task table columns to available IUs for execution. We further load balance between different task dividers by monitoring the last scheduled segment index in their task table, to approximately ensure similar progress among them.

Overheads of task dividers. The area of a task divider is mostly on the two tables, which are simply registers of a few hundreds of bytes. The latencies of each individual task divider and their coordination do not dominate the pipeline stages. The set operations on the IUs take relatively long time proportional to the entire set sizes, while the task dividers only work with the head lists that are shorter by a factor of $s_{l}$ or $s_{s}$.

\subsection{Input Distribution and Result Aggregation}

By leveraging segment-level parallelism, we distribute a single operation onto multiple IUs with the help of the task dividers in Section 4.2. To avoid excessive data bandwidth of transferring the relatively large and different segments to all IUs simultaneously, the $\mathrm{PE}$ distributes the input data in a round-robin fashion, one segment at a time, multicast to all IUs that require it. If the next IU has not finished its last workload, input data distribution would stall. Such a design is not a performance bottleneck, as it matches with the sequential processing style of the IUs, which also takes many cycles for set operations (see below).

On the output side, these separately computed results must be aggregated with caution. For example, assume a subtraction in Equation (1), where we subtract the long set from the short set. A short segment $\{11,18\}$ is paired with two overlapped long segments $\{3,5,7,12\}$ and $\{13,15,18,22\}$. The differences are $\{11,18\}$ and $\{11\}$. The final result should be the common subset, $\{11\}$, which requires another round of intersection, potentially across many IUs. This issue also exists for anti-subtraction if multiple short segments pair with one long segment.

FINGERS resolves this difficulty with an efficient and unified solution for all three set operations. Note that with two sets $A$ and $B$, $A-B=A-(A \cap B)$. We always compute the intersection of the two input segments on an IU regardless of the required operation, using a simple compare unit that streams in the two input lists similar to previous work [11, 47], as illustrated in Figure 8. The result is stored as a bitvector with the same length $s_{l}$ as the long segment, with different meanings for different operations. For intersection and anti-subtraction, the bitvector indicates whether each element in the long segment is in the intersection result; for subtraction (Figure 8), it indicates whether each element in the short segment is in the intersection result (padding with 1s).

To aggregate the results, we let the result collector sequentially receive the bitvector and its associated segment from each IU in a round-robin fashion, as shown in Figure 8. If the segment of the current IU does not match with the previous one, they belong to distinct ranges. We thus know that the previous segment has been fully collected and aggregated. It is now translated back to the list representation and concatenated with the existing result set. The new incoming segment stays in the result collector to wait for the next result.

Otherwise, if the same segment is received by the result collector, we need to aggregate the two bitvectors with bitwise OR. For intersection this is intuitive as we keep both results because $A \cap\left(B_{1} \cup B_{2}\right)=\left(A \cap B_{1}\right) \cup\left(A \cap B_{2}\right)$. For (anti-)subtraction, the results are the elements with $0 \mathrm{~s}$ in the bitvector, as the set difference 


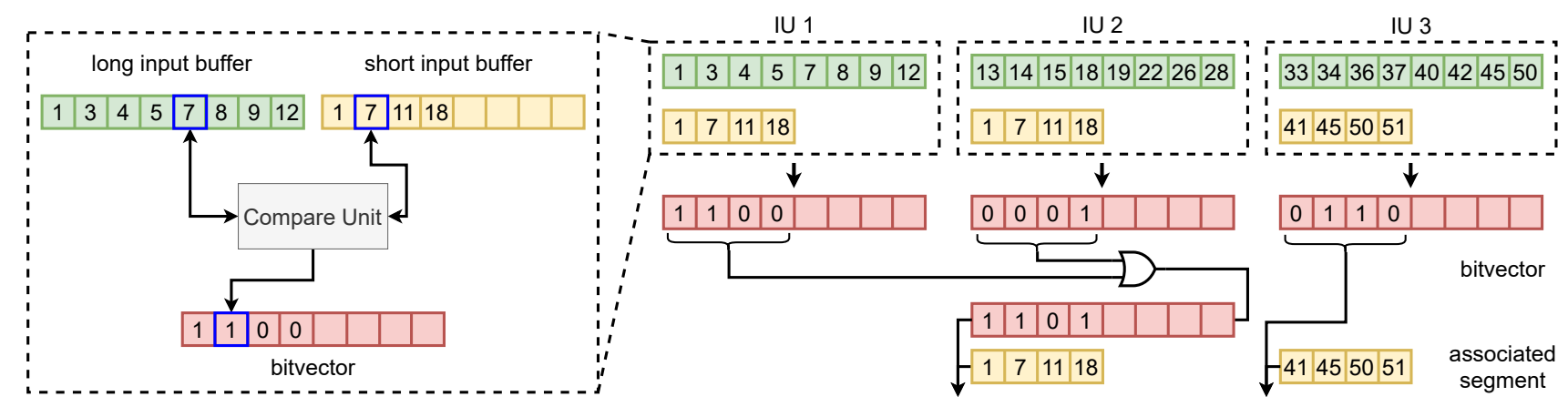

Figure 8: Computations in one intersect unit (IU) and result aggregation across IUs. The example is for subtraction.

Table 1: Evaluated real-world graph datasets.

\begin{tabular}{rrrrr}
\hline Dataset & \# Vertices & \# Edges & Avg Deg & Max Deg \\
\hline AstroPh (As) [34] & $18.8 \mathrm{~K}$ & $198 \mathrm{~K}$ & 21.1 & 504 \\
Mico (Mi) [17] & $80.0 \mathrm{~K}$ & $432 \mathrm{~K}$ & 10.8 & 936 \\
Youtube (Yo) [34] & $1.1 \mathrm{M}$ & $3.0 \mathrm{M}$ & 5.3 & 28,754 \\
Patents (Pa) [23] & $3.8 \mathrm{M}$ & $16.5 \mathrm{M}$ & 8.8 & 793 \\
LiveJournal (Lj) [34] & $4.8 \mathrm{M}$ & $42.9 \mathrm{M}$ & 17.7 & 20,333 \\
Orkut (Or) [34] & $3.1 \mathrm{M}$ & $117.2 \mathrm{M}$ & 76.3 & 33,313 \\
\hline
\end{tabular}

is the complement of the set intersection. Because $A-B_{1}-B_{2}=$ $\left(A-B_{1}\right) \cap\left(A-B_{2}\right)$, we need to keep the elements with 0 s in both bitvectors, i.e., again using bitwise OR.

In summary, both input distribution and result aggregation are done in a round-robin fashion among IUs. Both these serial time periods are proportional to the number of IUs in the PE. If this number is smaller than the cycles needed to perform set operations inside the IU, the input/output latencies can be well hidden by the computations. This is indeed the case with FINGERS, where we use 24 IUs per PE, and the IU processing time for one long segment paired with two or three short segments is about $s_{l}+3 \times s_{s}=28$.

\section{METHODOLOGY}

Benchmarks. We evaluate FINGERS with six commonly used patterns: 3- (i.e., triangle), 4-, and 5-clique (tc, 4cl, 5cl), tailed triangle $(t t)$, 4-cycle (cyc), and diamond (dia). We also evaluate a multipattern mining task, 3-motif $(3 \mathrm{mc})$, which demonstrates the generality of FINGERS. We use six real-world graphs, as listed in Table 1. All input graphs are undirected with no self-loop or duplicated edges.

Baselines. We mainly compare FINGERS with the latest, stateof-the-art graph mining accelerator, FlexMiner [11]. The two designs have the similar high-level architecture which allows for direct and fair comparisons. FlexMiner has been demonstrated an order of magnitude faster than the CPU baselines AutoMine [39] and GraphZero [38], as well as the prior hardware design Gramer [57].

Implementations and configurations. We implement all the newly added components in the FINGERS PE design in Verilog and synthesize using Synopsis DC in $28 \mathrm{~nm}$ to estimate the area and power, as reported in Section 6.1. The SRAM caches are modeled using CACTI [3]. We further implement a cycle-accurate simulator to evaluate the performance of FINGERS. Following the same configurations of FlexMiner, we use a $4 \mathrm{MB}$ shared cache, with four channels of DDR4-2666 off-chip DRAM that provide $85 \mathrm{~GB} / \mathrm{s}$. By default, each FINGERS PE uses 24 IUs, 12 task dividers, and a $32 \mathrm{kB}$ private cache. There are also two $8 \mathrm{kB}$ streaming buffers in front of the IUs to temporally store the segments to be processed. The overall FINGERS chip uses 20 PEs in order to keep iso-area with FlexMiner (see Section 6.1).

The same simulator is also used to reproduce the results for our baseline FlexMiner. This is fair as the high-level architecture is quite similar between the two, so we can just tune the concrete $\mathrm{PE}$ designs. We validate that the performance trends match with the results reported in the FlexMiner paper [11]. One noticeable difference is the c-map module in FlexMiner, which FINGERS does not use. This is due to the different algorithmic details in the two accelerators. FlexMiner used the c-map module to cache the union of neighbor lists of all vertices along the path from the search tree root to the current task [9]. In contrast, FINGERS aggregates these neighbor lists into the partially materialized candidate vertex sets $\left(S_{j(i)}\right)$ [39] and caches them in the PE private cache using similar or smaller spaces. Therefore, c-map is unnecessary in FINGERS.

We use the same execution plans when executing the benchmarks on both architectures, including vertex orders, set operation schedules, and symmetric breaking restrictions (see Section 2.1). Such information is generated by a compiler similar to that proposed in FlexMiner [11], which is orthogonal to our architectural improvements.

\section{EVALUATION}

In this section, we first report the synthesized results from the RTL implementation of a single FINGERS PE. Then we demonstrate the performance improvements of FINGERS using both single-PE and multi-PE configurations. Finally we study the detailed impacts of each individual parallelism level.

\subsection{PE Area, Power, and Frequency}

Table 2 breaks down the area consumption of a single FINGERS $\mathrm{PE}$, which is about $0.9 \mathrm{~mm}^{2}$ in $28 \mathrm{~nm}$. We conservatively infer the "Others" part, including control logic, NoC interface, and data fetchers, by scaling from FlexMiner. We can see that the 24 IUs only 
Table 2: Area breakdown of one FINGERS PE.

\begin{tabular}{rcr}
\hline Components & Area $\left(\mathrm{mm}^{2}\right)$ & \% Area \\
\hline 24 Intersect Units & 0.115 & $12.3 \%$ \\
12 Task Dividers & 0.069 & $7.4 \%$ \\
2 Stream Buffers & 0.214 & $22.9 \%$ \\
Private Cache & 0.118 & $12.6 \%$ \\
Others & 0.418 & $44.8 \%$ \\
\hline PE Total & 0.934 & $100 \%$ \\
\hline
\end{tabular}

contribute to a small portion of the area, and each IU takes only $0.005 \mathrm{~mm}^{2}$. The task dividers are also cheap components. Most of the area is spent on the local caching buffers and the control logic. Such results validate our design principle that scaling a PE into fine-grained parallel processing incurs little extra cost.

FlexMiner uses a PE of $0.18 \mathrm{~mm}^{2}$ under $15 \mathrm{~nm}$. When scaled to the same technology, the FINGERS PE $\left(0.26 \mathrm{~mm}^{2}\right.$ in $\left.15 \mathrm{~nm}\right)$ is less than twice large as the FlexMiner PE, despite the much higher processing throughput (up to $13.2 \times$, Section 6.2).

One FINGERS PE consumes $98.5 \mathrm{~mW}$ power on the compute logic and $85.6 \mathrm{~mW}$ on the caches. With 20 PEs per chip (see below), the total power of FINGERS would be just a few watts. FlexMiner did not report power results so we cannot directly compare, but it is likely the two behave similarly.

Our PE achieves $1 \mathrm{GHz}$ frequency in $28 \mathrm{~nm}$. We suspect it could match the $1.3 \mathrm{GHz}$ of FlexMiner after scaled to $15 \mathrm{~nm}$.

\subsection{Single PE Performance}

We first compare the single-PE performance between FINGERS and FlexMiner, to demonstrate that exploiting fine-grained parallelism within each PE can greatly improve performance. Figure 9 shows the results. The FINGERS PE outperforms the FlexMiner PE by $6.2 \times$ on average and up to $13.2 \times$ over all seven patterns and six graphs. We observe that different patterns and different graphs exhibit quite different speedups. This is mainly because of the different available types and drastically varying amounts of fine-grained parallelism that FINGERS can leverage in these benchmarks. We discuss the details below. Such diverse and irregular distribution necessitates FINGERS to simultaneously support all levels of finegrained parallelism.

Impacts of patterns. Clique counting does not have set-level parallelism as the candidate vertex sets for all future levels are always identical, so tc, 4cl, and $5 \mathrm{cl}$ show lower improvements than the other patterns. Larger clique patterns use deeper search trees. The branch-level parallelism decreases as the tree goes deeper, because fewer vertices can be valid candidates that connect to all ancestors. This reduces the benefits of FINGERS in As, $\mathrm{Pa}, \mathrm{Yo}$, and Or when the clique size increases, but not for $L j$ which has more large cliques and actually sees better performance. This is because the lower tree levels have better on-chip data reuse, as their demanded neighbor lists may already be fetched into the shared cache by the ancestors.

The $t t, c y c$, and dia patterns generally see the highest speedups among all patterns. This is because their set operation schedules involve (anti-)subtractions to produce large sets, which result in high parallelism that FINGERS can exploit. In particular, tt usually has large $S_{3(2)}$ (Figure 2), which leads to abundant segment-level parallelism when using it to calculate different $S_{3}$ later. cyc generates large $S_{2}$, which leads to high branch-level parallelism when used as the candidate vertex set for the next level. On the other hand, the subtraction operations in dia are only at the lower tree levels, where the sets are already shrunk small, so it shows lower benefits than the other two.

These three patterns all have size of 4 vertices, same as $4 \mathrm{cl}$. Their performance differences are attributed to the symmetric breaking restrictions. $4 \mathrm{cl}$ has more connections and thus more restrictions, reducing valid vertices and hence tasks to process.

Finally, 3mc is a multi-pattern task mining both triangles and wedges, which incurs more workloads than the single pattern of tc, and thus exhibits higher speedups.

Impacts of graphs. As and $\mathrm{Mi}$ are small graphs that all fit in the on-chip shared cache (see their tiny cache miss rates in Figure 13), so they enjoy higher improvements from the stronger PE design. As is actually so small that there are only a few embeddings matched with each pattern, so the independent workloads are limited. Mi has more cliques and thus even higher speedups. These small graphs are used to evaluate the on-chip parallelism and load balance capabilities.

On the other hand, Yo generally has the lowest speedups because of its lowest average degree (Table 1), which results in short neighbor lists and small sets, limiting the overall available parallelism for FINGERS. Yo is particularly good with tt and cyc, in which cases its candidate vertex sets are large (see above). Pa has very few high-degree vertices (i.e., low maximum degree, Table 1) and hence also limited benefits.

In contrast, $L j$ and Or are both very large graphs that exceed the on-chip cache size and are able to stress our memory system (Figure 13). They contain rich structures with many embedding matches, and thus exhibit abundant parallelism. Or has similar performance to $L j$ except for the patterns of large cliques, because it has fewer dense vertex clusters.

\subsection{Overall Performance}

With the additional coarse-grained parallelism at the PE level, we next demonstrate the overall performance improvements of FINGERS over FlexMiner in Figure 10. Since a FINGERS PE uses less than twice the area of a FlexMiner PE, for an iso-area evaluation we compare a 20-PE FINGERS chip with the 40-PE FlexMiner chip, which is its largest configuration used in the original paper [11]. Overall, FINGERS achieves on average $2.8 \times$ and up to $8.9 \times$ speedups over the baseline.

The general trends across patterns closely follow those in the single-PE setting as Figure 9. The impacts of input graphs become more significant. As mentioned above, As and Mi are small graphs fitting in the shared cache, so they see good scalability with more PEs. Their speedups are roughly half of those in Figure 9 due to the half number of PEs, but still reach $4.2 \times$ on average. Yo and $\mathrm{Pa}$ are large graphs with most vertices having low degrees, leading to large amount of data but limited work, especially on tc, 4cl, and $5 \mathrm{cl}$. So their memory access latency cannot be fully hidden by computations even with the help of branch-level parallelism in FINGERS, leading to limited speedups. Although $L j$ and $O r$ are 


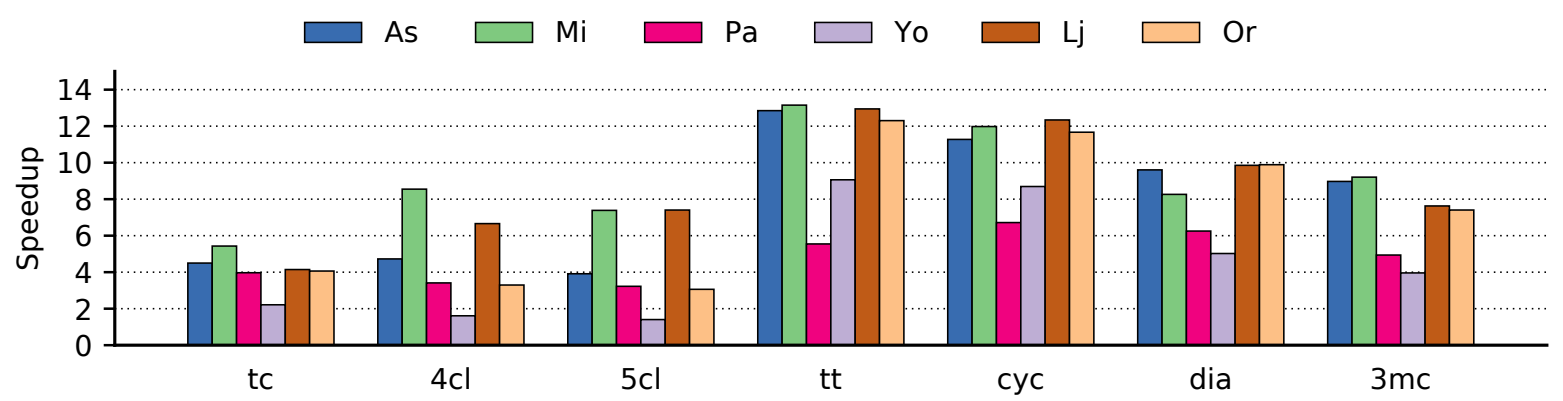

Figure 9: Single-PE speedups of FINGERS over FlexMiner.

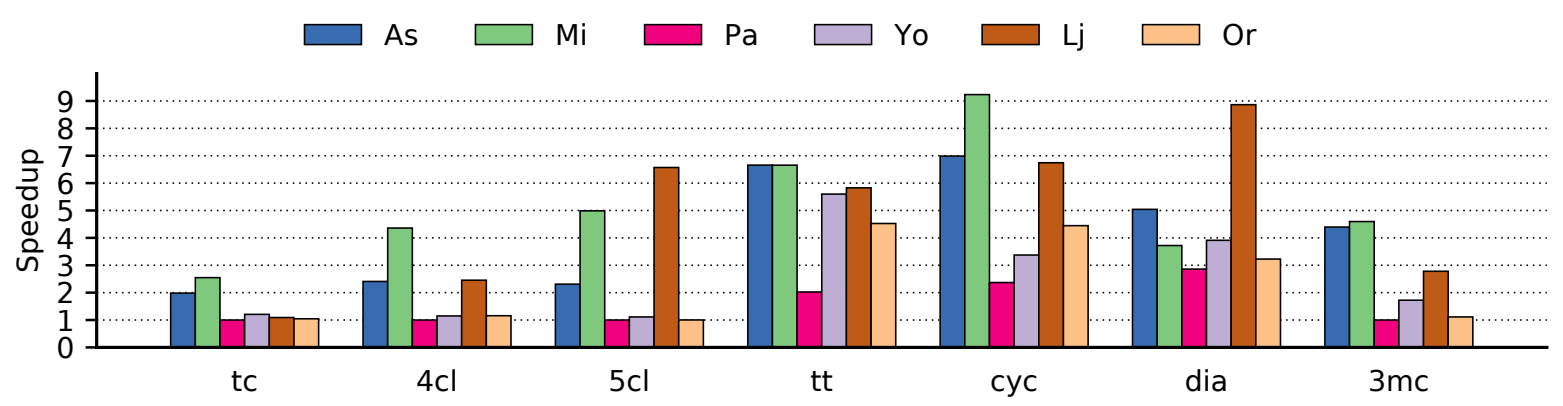

Figure 10: Overall speedups of FINGERS with 20 PEs over FlexMiner with 40 PEs.

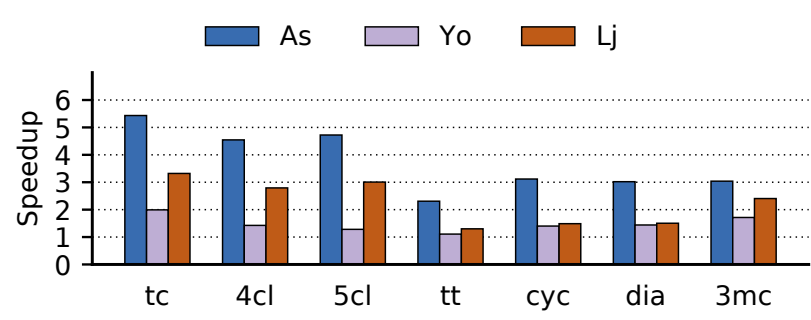

Figure 11: Speedups from branch-level parallelism and pseudo-DFS order.

also large, they exhibit more parallelism due to higher degrees, and thus their speedups are higher. One orthogonal way to improve memory access performance would be to simultaneously schedule search trees with nearby starting root vertices on different PEs, so they access similar vertices with high locality in the shared cache. Alternatively, FINGERS can be combined with processingin-memory technologies [4]. We leave this study as future work.

\subsection{In-Depth Studies}

Figure 11 presents the performance differences when enabling and disabling the pseudo-DFS order used in FINGERS (Section 4.1), to reflect the impacts of the branch-level parallelism. We present the results of three graphs; $\mathrm{Mi}, \mathrm{Pa}$, Or are similar to As, Yo, $L j$, respectively. We see that leveraging branch-level parallelism in

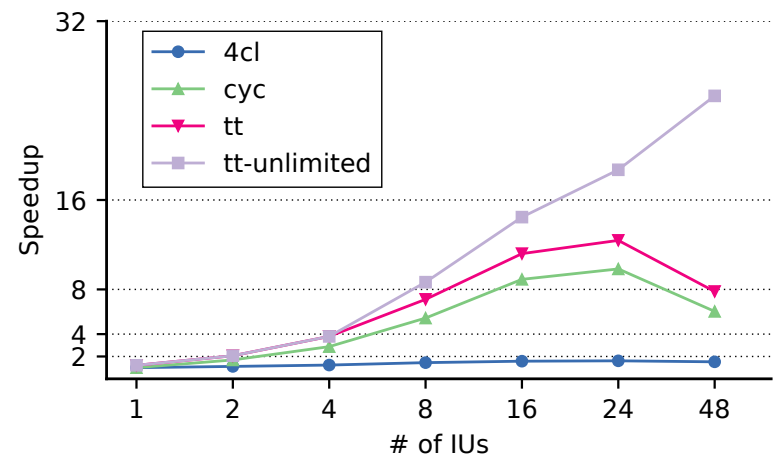

Figure 12: PE scalability in terms of numbers of IUs with Yo.

FINGERS provides up to $5 \times$ performance gains. The $\mathrm{tc}, 4 \mathrm{cl}$, and $5 \mathrm{cl}$ patterns benefit particularly significantly, because of their limited set-level and segment-level parallelism (see Section 6.2). Branchlevel parallelism is the major chance they can leverage.

Figure 12 studies the scalability of the FINGERS PE in terms of number of IUs, which closely relates to the utilization of setlevel and segment-level parallelism. Because using more IUs also increases area, we use an iso-area scaling approach, by keeping the product of the number of IUs and the long segment length constant, i.e., \# IUs $\times s_{l} \equiv 24 \times 16$. We show three patterns with the challenging Yo graph whose benefits are among the worst. 


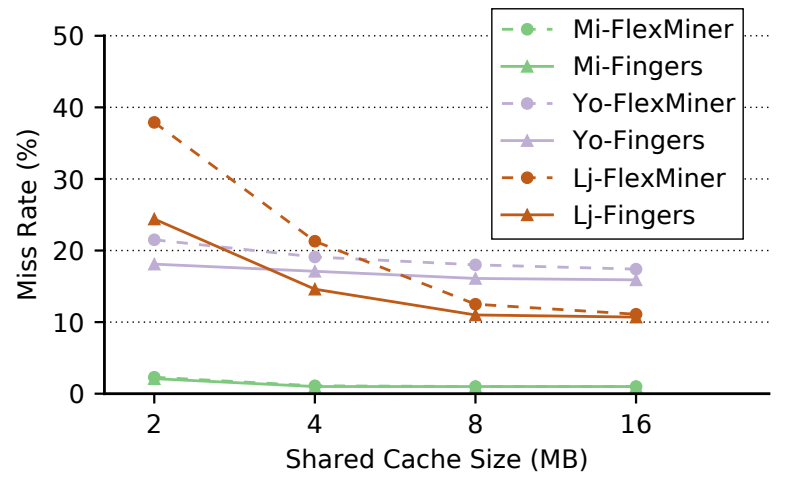

Figure 13: Shared cache miss curves (miss rate vs. capacity).

Table 3: IU utilization and load balance in one PE with Mi.

\begin{tabular}{rccccccc}
\hline & tc & $4 c 1$ & $5 c 1$ & tt & cyc & dia & $3 \mathrm{mc}$ \\
\hline Active Rate & $55.3 \%$ & $80.8 \%$ & $81.5 \%$ & $94.7 \%$ & $89.9 \%$ & $88.9 \%$ & $65.6 \%$ \\
Balance Rate & $67.3 \%$ & $66.4 \%$ & $66.3 \%$ & $68.2 \%$ & $70.3 \%$ & $71.4 \%$ & $69.3 \%$ \\
\hline
\end{tabular}

tt and cyc exhibit good scalability until 16 to 24 IUs, and the performance drops at 48 IUs. This is limited by area. If we allow unlimited area, the performance can further improve, as shown in tt-unlimited. In contrast, $4 \mathrm{cl}$ has poor scalability. The reason is still its limited set/segment-level parallelism, and it should use branchlevel parallelism instead (Figure 11). These results again motivate FINGERS to exploit all three levels of fine-grained parallelism.

To better understand the memory and shared cache behaviors in FINGERS, Figure 13 varies the shared cache capacity (default at $4 \mathrm{MB}$ ) and measures its miss rates when running the cyc pattern with three different graphs, using the same configurations as in Section 6.3. Mi (As similarly) is a small graph that fits in the cache, so its miss rates are consistently low in both FINGERS and FlexMiner. Although Yo ( $\mathrm{Pa}$ similarly) is large and constantly requires memory accesses, it has a small average vertex degree (Table 1) that allows most neighbor lists to stay in the shared cache for multiple times of reuse by the requesting PE, before being evicted by other neighbor lists. So the miss behaviors are similar in both designs and insensitive to the cache capacity. In contrast, $L j$ (Or similarly) is a large graph and has long neighbor lists, so it exhibits higher cache pressure. FINGERS more efficiently uses the shared cache with lower miss rates than FlexMiner, because it has fewer individual PEs competing for capacity. The pseudo-DFS order also decreases the miss rates by prioritizing the tasks whose data are already in the cache. In addition, with set-level parallelism, a long neighbor list could be streamed only once from memory to a PE and simultaneously reused by multiple candidate vertex sets (Figure 3 ), without polluting the small PE private cache as in FlexMiner. These trends validate our analysis of different graphs in Sections 6.2 and 6.3 .

Finally, Table 3 lists the utilization and the load balance behavior of the IUs in a PE, with the default 24 IUs. The active rate is the percentage of clock cycles during which workloads are assigned to the IUs. Recall that we assign each compute load to a subset of IUs
(Section 4.2). Within these cycles on each subset of IUs that execute a compute load, we further study load balance using the balance rate, which is the sum of the individual IU active cycles divided by the total cycle of this load times the subset size. For example, assuming 4 IUs, and only 2 IUs are assigned a load executed for 10 cycles. Then in a 20 -cycle period, the active rate is $25 \%$. If in those 10 cycles, one IU is fully used but the other is only active for 5 cycles, then the balance rate is only $75 \%$. We can see that in general the utilization is high (well exploiting branch-level parallelism) and the load balance is good (well exploiting set/segment-level parallelism).

\section{CONCLUSIONS}

In this paper we propose FINGERS, a novel graph mining accelerator that exploits fine-grained parallelism at the branch, set, and segment levels during search tree exploration and set operations of pattern-aware graph mining. FINGERS efficiently handles resource allocation, task scheduling, load balancing, and input/output communication with a pipelined processing element architecture enhanced with multiple compute units. Compared with a stateof-the-art baseline that only utilizes coarse-grained parallelism, FINGERS achieves up to $8.9 \times$ performance improvements at the same chip area, and benefits from different parallelism levels in different patterns and graphs.

\section{ACKNOWLEDGMENTS}

The authors thank the anonymous reviewers and our shepherd, Johnathan Alsop, for their valuable suggestions. This work was supported by the National Natural Science Foundation of China (62072262). Mingyu Gao is the corresponding author.

\section{REFERENCES}

[1] Nesreen K Ahmed, Jennifer Neville, Ryan A Rossi, and Nick Duffield. 2015. Efficient Graphlet Counting for Large Networks. In International Conference on Data Mining (ICDM). 1-10.

[2] Noga Alon, Phuong Dao, Iman Hajirasouliha, Fereydoun Hormozdiari, and S Cenk Sahinalp. 2008. Biomolecular Network Motif Counting and Discovery by Color Coding. Bioinformatics 24, 13 (2008), i241-i249.

[3] Rajeev Balasubramonian, Andrew B. Kahng, Naveen Muralimanohar, Ali Shafiee, and Vaishnav Srinivas. 2017. CACTI 7: New Tools for Interconnect Exploration in Innovative Off-Chip Memories. ACM Transactions on Architecture and Code Optimization 14, 2 (2017).

[4] Maciej Besta, Raghavendra Kanakagiri, Grzegorz Kwasniewski, Rachata Ausavarungnirun, Jakub Beránek, Konstantinos Kanellopoulos, Kacper Janda, Zur Vonarburg-Shmaria, Lukas Gianinazzi, Ioana Stefan, Juan Gómez Luna, Jakub Golinowski, Marcin Copik, Lukas Kapp-Schwoerer, Salvatore Di Girolamo, Nils Blach, Marek Konieczny, Onur Mutlu, and Torsten Hoefler. 2021. SISA: SetCentric Instruction Set Architecture for Graph Mining on Processing-in-Memory Systems. In 54th Annual IEEE/ACM International Symposium on Microarchitecture (MICRO). 282-297.

[5] Bibek Bhattarai, Hang Liu, and H Howie Huang. 2019. CECI: Compact Embedding Cluster Index for Scalable Subgraph Matching. In 2019 International Conference on Management of Data (SIGMOD). 1447-1462.

[6] Fei Bi, Lijun Chang, Xuemin Lin, Lu Qin, and Wenjie Zhang. 2016. Efficient Subgraph Matching by Postponing Cartesian Products. In 2016 International Conference on Management of Data (SIGMOD). 1199-1214.

[7] Hongzhi Chen, Miao Liu, Yunjian Zhao, Xiao Yan, Da Yan, and James Cheng. 2018. G-Miner: An Efficient Task-Oriented Graph Mining System. In 13th European Conference on Computer Systems (EuroSys). 1-12.

[8] Jingji Chen and Xuehai Qian. 2020. DwarvesGraph: A High-Performance Graph Mining System with Pattern Decomposition. arXiv preprint arXiv:2008.09682 (2020).

[9] Xuhao Chen, Roshan Dathathri, Gurbinder Gill, Loc Hoang, and Keshav Pingali. 2021. Sandslash: A Two-Level Framework for Efficient Graph Pattern Mining. In International Conference on Supercomputing (ICS). 378-391. 
[10] Xuhao Chen, Roshan Dathathri, Gurbinder Gill, and Keshav Pingali. 2020. Pangolin: An Efficient and Flexible Graph Mining System on CPU and GPU. Proceedings of the VLDB Endowment 13, 8 (2020), 1190-1205.

[11] Xuhao Chen, Tianhao Huang, Shuotao Xu, Thomas Bourgeat, and Chanwoo Chung. 2021. FlexMiner: A Pattern-Aware Accelerator for Graph Pattern Mining In 48th Annual International Symposium on Computer Architecture (ISCA). 581594

[12] Norishige Chiba and Takao Nishizeki. 1985. Arboricity and Subgraph Listing Algorithms. SIAM F. Comput. 14, 1 (1985), 210-223.

[13] Young-Rae Cho and Aidong Zhang. 2009. Predicting Protein Function by Frequent Functional Association Pattern Mining in Protein Interaction Networks. IEEE Transactions on Information Technology in Biomedicine 14, 1 (2009), 30-36.

[14] Maximilien Danisch, Oana Balalau, and Mauro Sozio. 2018. Listing k-cliques in Sparse Real-World Graphs. In 2018 World Wide Web Conference (WWW). 589-598.

[15] Mukund Deshpande, Michihiro Kuramochi, Nikil Wale, and George Karypis. 2005. Frequent Substructure-Based Approaches for Classifying Chemical Compounds. IEEE Transactions on Knowledge and Data Engineering 17, 8 (2005), 1036-1050.

[16] Vinicius Dias, Carlos HC Teixeira, Dorgival Guedes, Wagner Meira, and Srinivasan Parthasarathy. 2019. Fractal: A General-Purpose Graph Pattern Mining System. In 2019 International Conference on Management of Data (SIGMOD). 13571374.

[17] Mohammed Elseidy, Ehab Abdelhamid, Spiros Skiadopoulos, and Panos Kalnis. 2014. Grami: Frequent Subgraph and Pattern Mining in a Single Large Graph. Proceedings of the VLDB Endowment 7, 7 (2014), 517-528.

[18] Katherine Faust. 2010. A Puzzle Concerning Triads in Social Networks: Graph Constraints and the Triad Census. Social Networks 32, 3 (2010), 221-233.

[19] Ove Frank. 1988. Triad Count Statistics. Discrete Mathematics 72, 1 (1988), 141-149.

[20] Benoit Gaüzere, Luc Brun, and Didier Villemin. 2012. Two New Graphs Kernels in Chemoinformatics. Pattern Recognition Letters 33, 15 (2012), 2038-2047.

[21] Lukas Gianinazzi, Maciej Besta, Yannick Schaffner, and Torsten Hoefler. 2021 Parallel Algorithms for Finding Large Cliques in Sparse Graphs. In 33rd ACM Symposium on Parallelism in Algorithms and Architectures (SPAA). 243-253.

[22] Ilias Giechaskiel, George Panagopoulos, and Eiko Yoneki. 2015. PDTL: Parallel and Distributed Triangle Listing for Massive Graphs. In 44th International Conference on Parallel Processing (ICPP). 370-379.

[23] Bronwyn H Hall, Adam B Jaffe, and Manuel Trajtenberg. 2001. The NBER Patent Citation Data File: Lessons, Insights and Methodological Tools. Technical Report. National Bureau of Economic Research.

[24] Tae Jun Ham, Lisa Wu, Narayanan Sundaram, Nadathur Satish, and Margaret Martonosi. 2016. Graphicionado: A High-Performance and Energy-Efficient Accelerator for Graph Analytics. In 49th Annual IEEE/ACM International Symposium on Microarchitecture (MICRO). 1-13.

[25] Loc Hoang, Vishwesh Jatala, Xuhao Chen, Udit Agarwal, Roshan Dathathri, Gurbinder Gill, and Keshav Pingali. 2019. DistTC: High Performance Distributed Triangle Counting. In 2019 IEEE High Performance Extreme Computing Conference (HPEC). 1-7.

[26] Paul W Holland and Samuel Leinhardt. 1976. Local Structure in Social Networks. Sociological Methodology 7 (1976), 1-45.

[27] Yang Hu, Hang Liu, and H Howie Huang. 2018. TriCore: Parallel Triangle Counting on GPUs. In International Conference for High Performance Computing, Networking, Storage and Analysis (SC). 171-182.

[28] Hiroshi Inoue, Moriyoshi Ohara, and Kenjiro Taura. 2014. Faster Set Intersection with SIMD Instructions by Reducing Branch Mispredictions. Proceedings of the VLDB Endowment 8, 3 (2014), 293-304.

[29] Kasra Jamshidi, Rakesh Mahadasa, and Keval Vora. 2020. Peregrine: A PatternAware Graph Mining System. In 15th European Conference on Computer Systems (EuroSys). 1-16.

[30] Oren Kalinsky, Benny Kimelfeld, and Yoav Etsion. 2020. The TrieJax Architecture: Accelerating Graph Operations Through Relational Joins. In 25th International Conference on Architectural Support for Programming Languages and Operating Systems (ASPLOS). 1217-1231.

[31] Hisashi Kashima, Hiroto Saigo, Masahiro Hattori, and Koji Tsuda. 2011. Graph Kernels for Chemoinformatics. In Chemoinformatics and Advanced Machine Learning Perspectives: Complex Computational Methods and Collaborative Techniques. IGI Global, 1-15.

[32] Hyeonji Kim, Juneyoung Lee, Sourav S Bhowmick, Wook-Shin Han, JeongHoon Lee, Seongyun Ko, and Moath HA Jarrah. 2016. DUALSIM: Parallel Subgraph Enumeration in a Massive Graph on a Single Machine. In 2016 International Conference on Management of Data (SIGMOD). 1231-1245.

[33] Kyoungmin Kim, In Seo, Wook-Shin Han, Jeong-Hoon Lee, Sungpack Hong Hassan Chafi, Hyungyu Shin, and Geonhwa Jeong. 2018. TurboFlux: A Fast Continuous Subgraph Matching System for Streaming Graph Data. In 2018 International Conference on Management of Data (SIGMOD). 411-426.

[34] Jure Leskovec and Andrej Krevl. 2014. SNAP Datasets: Stanford Large Network Dataset Collection.
[35] Chenhao Ma, Reynold Cheng, Laks VS Lakshmanan, Tobias Grubenmann, Yixiang Fang, and Xiaodong Li. 2019. LINC: A Motif Counting Algorithm for Uncertain Graphs. Proceedings of the VLDB Endowment 13, 2 (2019), 155-168.

[36] Shuai Ma, Yang Cao, Jinpeng Huai, and Tianyu Wo. 2012. Distributed Graph Pattern Matching. In 21st International Conference on World Wide Web $(W W W)$. 949-958.

[37] Grzegorz Malewicz, Matthew H Austern, Aart JC Bik, James C Dehnert, Ilan Horn, Naty Leiser, and Grzegorz Czajkowski. 2010. Pregel: A System for LargeScale Graph Processing. In 2010 International Conference on Management of data (SIGMOD). 135-146.

[38] Daniel Mawhirter, Sam Reinehr, Connor Holmes, Tongping Liu, and Bo Wu. 2021. GraphZero: A High-Performance Subgraph Matching System. ACM SIGOPS Operating Systems Review 55, 1 (2021), 21-37.

[39] Daniel Mawhirter and Bo Wu. 2019. AutoMine: Harmonizing High-Level Abstraction and High Performance for Graph Mining. In 27th ACM Symposium on Operating Systems Principles (SOSP). 509-523.

[40] Tijana Milenković, Weng Leong Ng, Wayne Hayes, and Nataša Pržulj. 2010. Optimal Network Alignment with Graphlet Degree Vectors. Cancer Informatics 9 (2010), CIN-S4744.

[41] Tijana Milenković and Nataša Pržulj. 2008. Uncovering Biological Network Function via Graphlet Degree Signatures. Cancer Informatics 6 (2008), CIN-S680.

[42] Lawrence Page, Sergey Brin, Rajeev Motwani, and Terry Winograd. 1999. The Pagerank Citation Ranking: Bringing Order to the Web. Technical Report. Stanford InfoLab.

[43] Santosh Pandey, Xiaoye Sherry Li, Aydin Buluc, Jiejun Xu, and Hang Liu. 2019. H-INDEX: Hash-Indexing for Parallel Triangle Counting on GPUs. In 2019 IEEE High Performance Extreme Computing Conference (HPEC). 1-7.

[44] Roger Pearce, Trevor Steil, Benjamin W Priest, and Geoffrey Sanders. 2019. One Quadrillion Triangles Queried on One Million Processors. In 2019 IEEE High Performance Extreme Computing Conference (HPEC). 1-5.

[45] Ali Pinar, C Seshadhri, and Vaidyanathan Vishal. 2017. ESCAPE: Efficiently Counting All 5-vertex Subgraphs. In 26th International Conference on World Wide Web $(W W W) .1431-1440$

[46] Liva Ralaivola, Sanjay J Swamidass, Hiroto Saigo, and Pierre Baldi. 2005. Graph Kernels for Chemical Informatics. Neural Networks 18, 8 (2005), 1093-1110.

[47] Gengyu Rao, Jingji Chen, Jason Yik, and Xuehai Qian. 2020. IntersectX: An Efficient Accelerator for Graph Mining, arXiv preprint arXiv:2012.10848 (2020).

[48] Amitabha Roy, Ivo Mihailovic, and Willy Zwaenepoel. 2013. X-Stream: EdgeCentric Graph Processing Using Streaming Partitions. In 24th ACM Symposium on Operating Systems Principles (SOSP). 472-488.

[49] Yingxia Shao, Bin Cui, Lei Chen, Lin Ma, Junjie Yao, and Ning Xu. 2014. Parallel Subgraph Listing in a Large-Scale Graph. In 2014 International Conference on Management of Data (SIGMOD). 625-636.

[50] Tianhui Shi, Mingshu Zhai, Yi Xu, and Jidong Zhai. 2020. GraphPi: High Performance Graph Pattern Matching Through Effective Redundancy Elimination. In International Conference for High Performance Computing, Networking, Storage and Analysis (SC). 1-14.

[51] Julian Shun and Guy E Blelloch. 2013. Ligra: A Lightweight Graph Processing Framework for Shared Memory. In 18th ACM SIGPLAN Symposium on Principles and Practice of Parallel Programming (PPoPP). 135-146.

[52] Shixuan Sun and Qiong Luo. 2019. Scaling Up Subgraph Query Processing with Efficient Subgraph Matching. In 35th International Conference on Data Engineering (ICDE). 220-231.

[53] Carlos HC Teixeira, Alexandre J Fonseca, Marco Serafini, Georgos Siganos, Mohammed J Zaki, and Ashraf Aboulnaga. 2015. Arabesque: A System for Distributed Graph Mining. In 25th ACM Symposium on Operating Systems Principles (SOSP). 425-440.

[54] Vasileios Trigonakis, Jean-Pierre Lozi, Tomáš Faltín, Nicholas P. Roth, Iraklis Psaroudakis, Arnaud Delamare, Vlad Haprian, Calin Iorgulescu, Petr Koupy, Jinsoo Lee, Sungpack Hong, and Hassan Chafi. 2021. aDFS: An Almost DepthFirst-Search Distributed Graph-Querying System. In USENIX Annual Technical Conference (ATC). 209-224.

[55] Kai Wang, Zhiqiang Zuo, John Thorpe, Tien Ouang Nguyen, and Guoqing Harry Xu. 2018. RStream: Marrying Relational Algebra with Streaming for Efficient Graph Mining on a Single Machine. In 13th USENIX Symposium on Operating Systems Design and Implementation (OSDI). 763-782.

[56] Da Yan, Guimu Guo, Md Mashiur Rahman Chowdhury, M Tamer Özsu, WeiShinn Ku, and John CS Lui. 2020. G-thinker: A Distributed Framework for Mining Subgraphs in a Big Graph. In 36th International Conference on Data Engineering (ICDE). 1369-1380.

[57] Pengcheng Yao, Long Zheng, Zhen Zeng, Yu Huang, Chuangyi Gui, Xiaofei Liao, Hai Jin, and Jingling Xue. 2020. A Locality-Aware Energy-Efficient Accelerator for Graph Mining Applications. In 53rd Annual IEEE/ACM International Symposium on Microarchitecture (MICRO). 895-907.

[58] Olaf Zorzi. 2019. Granovetter (1983): The Strength of Weak Ties: A Network Theory Revisited. Springer Fachmedien Wiesbaden, 243-246. 